\title{
Interaction of Salmonella choleraesuis, Salmonella dublin and Salmonella typhimurium with porcine and bovine terminal ileum in vivo
}

\author{
Alex J. Bolton, ${ }^{1}+$ Michael P. Osborne, ${ }^{2}$ Tim S. Wallis ${ }^{3}$ and John Stephen ${ }^{1}$
}

Author for correspondence: John Stephen. Tel: +44 121414 6550. Fax: + 441214146557. e-mail: J.Stephen@bham.ac.uk

1,2 Molecular Microbiology and Cell Biology Group, School of Biological Sciences ${ }^{1}$ and Department of Physiology, The Medical School2, University of Birmingham, Edgbaston, Birmingham B15 2TT, UK

3 Institute for Animal Health, Compton, Newbury RG20 7NN, UK
Quantitative experiments on the interaction of Salmonella choleraesuis and Salmonella dublin with porcine and bovine intestinal epithelia yielded no evidence to suggest that host restriction of $S$. choleraesuis and $S$. dublin for pigs and calves respectively could be explained in terms of the patterns of intestinal invasion observed in ligated ileal loops in vivo, at $3 \mathbf{~ h}$ after challenge. No evidence was found to support the idea that Peyer's patches, or specifically $M$ cells, are the major route of entry for these serotypes in vivo. Three hours after loop inoculation, each serotype was recovered in comparable numbers from either absorptive or Peyer's patch mucosae present in the same ileal loop, indicating that both types of tissue are involved in the early stages of the enteropathogenic process induced by both serotypes. More detailed transmission electron microscopic (TEM) analyses of follicle-associated epithelia (FAE) challenged with S. choleraesuis showed that in the same region of FAE, organisms invaded both $M$ cells and enterocytes directly; comparable detailed TEM studies with $S$. dublin could not be carried out because of the tissue-destructive properties of this serotype. S. dublin was clearly more histotoxic than S. choleraesuis as had previously been found in rabbits: this difference is almost certainly due to a tissue-damaging toxin which is neither host nor gut-tissue specific. The tissue-destructive potential of $S$. dublin has profound implications for the measurement of and the assignment of significance to the invasiveness of $S$. dublin. S. dublin was nearly always seen entering gut cells in micro-colonies whereas $S$. choleraesuis entered mainly as single organisms or small groups of two or three.

Keywords: invasion, intestine, $\mathrm{M}$ cells, host restriction, histotoxicity

\section{INTRODUCTION}

Here we report comparative studies on the invasiveness of Salmonella choleraesuis and Salmonella dublin for porcine and bovine gut in vivo. The first objective of the study was to establish the patterns of invasiveness of $S$. dublin and S. choleraesuis in vivo by challenging both pigs and calves using the ligated ileal loop technique.

\footnotetext{
†Present address: Dept of Microbiology \& Infectious Diseases, University of Calgary Health Sciences Centre, Calgary, Alberta, Canada T2N 4N1.

Abbreviations: $A E$, absorptive epithelium; FAE, follicle-associated epithelium; PMN, polymorphonuclear leucocyte; TEM, transmission electron microscopy.
}

From such studies it was hoped to obtain data bearing on the question of 'host restriction' of these two serotypes. Could this be explained - at least in part - by differential susceptibility of pigs and cattle to initial intestinal invasion by these two Salmonella serotypes? In a previous study there was no significant difference in the initial invasiveness of $S$. choleraesuis, $S$. dublin and Salmonella typhimurium for rabbit gut under conditions that suppressed the histotoxic activity of $S$. dublin (Bolton et al., 1999). However, as was pointed out (Bolton et al., 1999), 'the final view as to whether host restriction is determined by the relative ease with which these serotypes invade their respective susceptible hosts must await comparative studies on porcine and bovine tissues'. Here we report the results of such experiments. 
Table 1. Bacterial strains used in this work

\begin{tabular}{|c|c|}
\hline Strain & Origin and characteristics \\
\hline S. choleraesuis A50 & $\begin{array}{l}\text { Var. kunzendorf; isolated from a case of salmonellosis in pigs } \\
\text { in the UK }\end{array}$ \\
\hline S. choleraesuis $14 / 74$ & $\begin{array}{l}\text { Var. kunzendorf; isolated from a case of salmonellosis in pigs } \\
\text { in the UK }\end{array}$ \\
\hline S. dublin 3246 & Isolated from a case of salmonellosis in calves in the UK \\
\hline S. dublin 2229 & Isolated from a case of salmonellosis in calves in the UK \\
\hline S. typhimurium $4 / 74$ & $\begin{array}{l}\text { Virulent invasive strain isolated from a case of salmonellosis } \\
\text { in calves in the UK (Watson et al., 1995); used as positive } \\
\text { invasive control }\end{array}$ \\
\hline S. typhimurium 4/74 invH & $\begin{array}{l}\text { invH201:: TnphoA; avirulent, less invasive isogenic mutant of } \\
\text { S. typhimurium } 4 / 74 \text { (Watson et al., 1995); used as negative } \\
\text { invasive control }\end{array}$ \\
\hline E. coli $\mathrm{B} 44$ & $\begin{array}{l}\text { Nalidixic-acid-resistant strain, adherent but non-invasive for } \\
\text { bovine intestinal epithelium (Watson } \text { et al., 1995); used to } \\
\text { validate efficiency of gentamicin killing of non-internalized } \\
\text { organisms }\end{array}$ \\
\hline
\end{tabular}

The second objective was to ascertain the portal of entry into porcine gut epithelium. Many studies of Salmonella/intestinal interactions are carried out in mice with strains of S. typhimurium, which have given rise to the perception that the portal of entry into the host for Salmonellae is via Peyer's patches, in particular M cells (Jones et al., 1994; Clark et al., 1994, 1996; Penheiter et al., 1997). However, we have shown that S. typhimurium (Wallis et al., 1986; Worton et al., 1989), and S. dublin and $S$. choleraesuis (Bolton et al., 1999), enter rabbit enterocytes directly. Two recent studies in calves (Frost et al., 1997; Watson et al., 1995) demonstrated that both follicle-associated epithelium (FAE), which primarily consists of both enterocytes and $\mathrm{M}$ cells, and absorptive epithelium (AE), which lacks $M$ cells, were rapidly invaded. An examination, by light and electron microscopy, of bovine ileal mucosa from loops infused with $S$. typhimurium showed that within $5 \mathrm{~min}$, organisms were interacting with and destroying $M$ cells lining domed villi associated with FAE; and by $1 \mathrm{~h}$, bacteria were seen in macrophages in the lamina propria. However, in absorptive villi, bacteria were also seen adhering to enterocytes by $15 \mathrm{~min}$; the process of bacterial internalization, exfoliation of bacteria-laden enterocytes and stunting of villi had begun by $30 \mathrm{~min}$, and by 2 h these changes were severe (Frost et al., 1997). In a quantitative in vivo study no differences were found in the numbers of tissue-associated S. typhimurium and $S$. dublin organisms recovered from bovine ileal loops, with or without Peyer's patches, $3 \mathrm{~h}$ after inoculation (Watson et al., 1995). Since the nature of the Salmonella/host interaction is both serotype- and hostdependent, we have now examined, by electron microscopy, porcine tissues challenged with S. dublin and $S$. choleraesuis to determine if entry into $M$ cells is a sine qua non for Salmonella invasion of ileal epithelia in pigs.

The third objective was to determine if the differential histotoxicity shown by $S$. dublin but not $S$. choleraesuis for rabbit tissue (Bolton et al., 1999; Lodge et al., 1999) was expressed in porcine and bovine tissue.

\section{METHODS}

Bacterial strains and cultural conditions. Details of the bacterial strains are listed in Table 1 . All cultural and storage conditions used were as described by Bolton et al. (1999). All strains were equally sensitive to gentamicin (Watson et al., 1995; Bolton et al., 1999).

Animals. Pigs were farm-reared Landrace/Large White cross male pigs 7-8 weeks of age. Pigs were weaned at 3 weeks and fed on Fastgrow pellets (without antibiotics) (Gro-well Feeds, Melksham, Wilts, UK). Calves were farm-reared Fresian bull calves $28 \mathrm{~d}$ old which were fed on powdered milk from $2 \mathrm{~d}$ old. Pigs and calves were obtained from the farms at the Institute for Animal Health, Compton.

Bovine/porcine ileal loop invasion assays. The surgical techniques were carried out as described previously (Watson et al., 1995). Animals were terminally anaesthetized with sodium pentabarbitone. Commencing at the distal ileum, a maximum of 14 sequential loops (each $9 \mathrm{~cm}$ in length and containing both AE and FAE) were ligated, separated by $1 \mathrm{~cm}$ loops. Approximately $10^{9}$ organisms, suspended in Hartley Digest Broth (HDB) medium (Oxoid), were injected into appropriate loops, with alternating strains in sequential loops. In a typical experiment, three loops were infected with $S$. choleraesuis, three loops were infected with S. dublin, one loop was infected with the invasive wild-type S. typhimurium strain $4 / 74$, one with its less invasive isogenic inv $H$ mutant, and one with HDB. The ligated loops were replaced inside the abdomen and the wound repaired. Following $2 \mathrm{~h}$ incubation, $4.5 \mathrm{ml}$ of $300 \mu \mathrm{g}$ gentamicin $\mathrm{ml}^{-1}$ in mucosal medium (MM; Amin et al., 1994) was injected into each loop. After a further $1 \mathrm{~h}$ incubation, designated loops were fixed for electron microscopy, or biopsies were taken for quantitative bacterial viable counts as follows.

Loops were placed immediately into ice-cold MM and washed in the same medium. Three biopsies of AE and three biopsies of FAE were taken from each loop with a $12 \mathrm{~mm}$ cork borer and homogenized separately for $30 \mathrm{~s}$ in $3 \mathrm{ml}$ of $1 \%(\mathrm{v} / \mathrm{v})$ Triton X-100 (Sigma) in phosphate-buffered saline $(\mathrm{NaCl}$, 


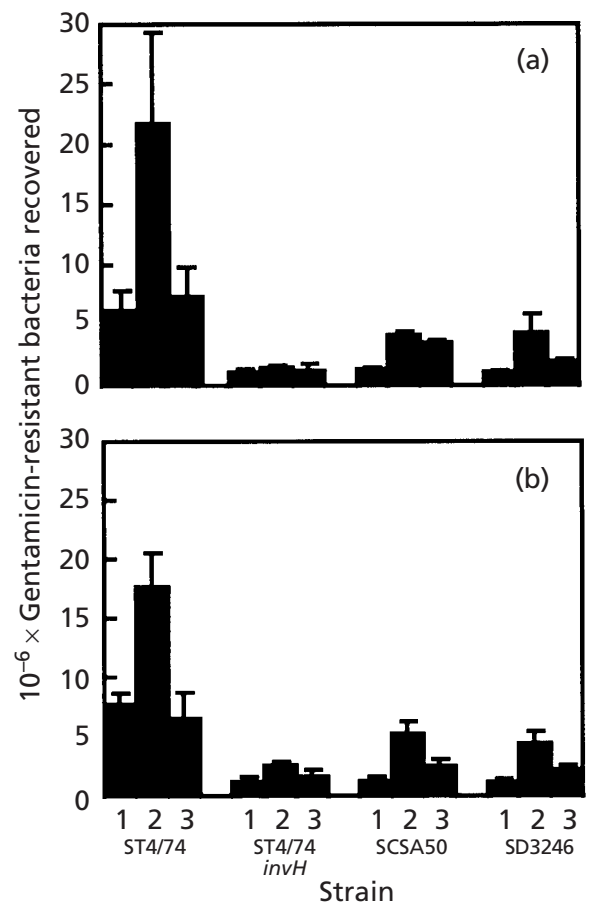

Fig. 1. Recoveries of S. choleraesuis A50 (SCSA50) and S. dublin 3246 (SD3246) from porcine ileal invasion assays in ligated loops in vivo. Each strain was tested in three pigs (designated 1, 2 and 3; horizontal axes). In each pig, three loops were used for $S$. choleraesuis A50 and three for S. dublin 3246. From each loop, after gentamicin treatment, three biopsies of $A E(a)$ and three biopsies of FAE (b) were taken, homogenized and viable counts made. Each column represents the mean and standard error of the mean of numbers of gentamicin-resistant bacteria recovered from nine biopsies each for $S$. choleraesuis $A 50$ and $S$. dublin 3246 taken from one pig. S. typhimurium strain 4/74 (ST4/74) and its isogenic invH mutant (ST4/74 invH) were used as positive and negative controls respectively. Since the relative invasiveness of these strains had already been quantified in calves (Watson et al., 1995), only one loop, and three biopsies, were used for each control strain in pigs (Fig. 1) and calves (Fig. 2).

$8.0 \mathrm{~g} ; \mathrm{KCl}, 0.2 \mathrm{~g} ; \mathrm{Na}_{2} \mathrm{HPO}_{4}, 1.43 \mathrm{~g}$; and $\mathrm{KH}_{2} \mathrm{PO}_{4}, 0.2 \mathrm{~g}$ dissolved and made up to 1 litre with distilled water; $\mathrm{pH}$ adjusted to $7 \cdot 3$ with $\mathrm{HCl}$ ). Serial dilutions were prepared for each sample and plated in triplicate on to both MacConkey and Brilliant green agar and incubated overnight at $37^{\circ} \mathrm{C}$. Brilliant green agar was used to suppress growth of indigenous Escherichia coli. Counts were made of the number of viable, i.e. gentamicin-resistant, organisms recovered per sample. The numbers of viable gentamicin-resistant bacteria recovered were used as a measure of invasiveness.

Sample tissues became available for electron microscopic analyses only, from one pig in which exposure to challenge was $90 \mathrm{~min}$. In this experiment, two additional strains were used: S. dublin 2229 and S. choleraesuis 14/74.

The effectiveness of gentamicin killing. Loops containing either AE or FAE were challenged with approximately $10^{9}$ adherent non-invasive E. coli B44; control loops were injected with MM. After $1 \mathrm{~h}$ incubation, $4.5 \mathrm{ml}$ gentamicin $(300 \mu \mathrm{g}$ $\mathrm{ml}^{-1}$ in MM) was injected into loops; $4.5 \mathrm{ml} \mathrm{MM}$ was injected as control. By this means it was established that $>99 \%$ of the organisms were killed.

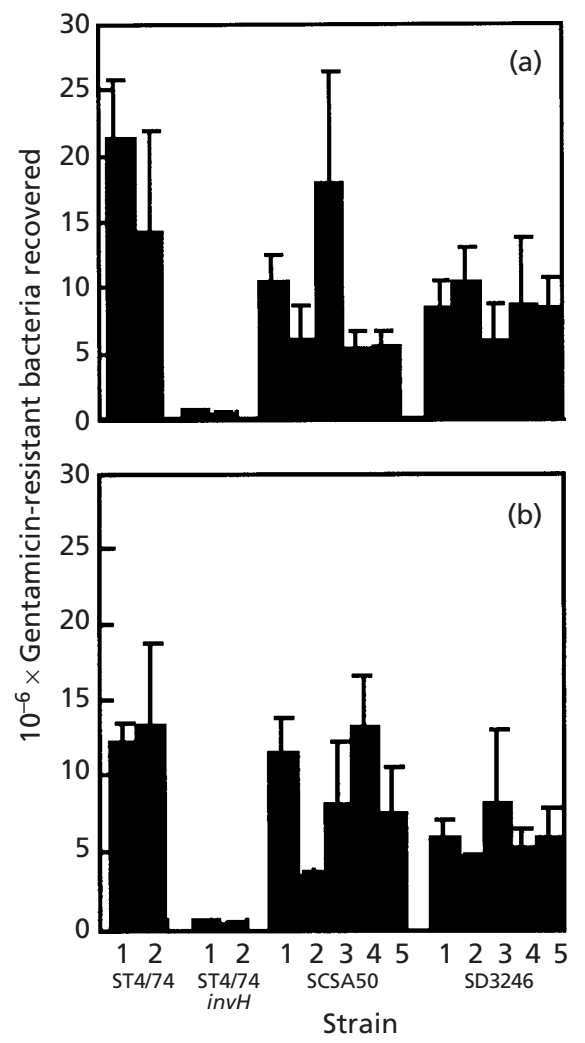

Fig. 2. Recoveries of $S$. choleraesuis $A 50$ (SCSA50) and S. dublin 3246 (SD3246) from bovine ileal invasion assays in ligated loops in vivo. Five calves were used (designated 1, 2, 3, 4 and 5; horizontal axes). All four strains were tested in calves 1 and 2; only S. choleraesuis A50 and S. dublin 3246 were tested in calves 3, 4 and 5 . In each calf, three loops were used for $S$. choleraesuis A50 and three for S. dublin 3246. From each loop, after gentamicin treatment, three biopsies of $A E(a)$ and three biopsies of FAE (b) were taken, homogenized and viable counts made. Each column represents the mean and standard error of the mean of numbers of gentamicin-resistant bacteria recovered from 9 biopsies each for S. choleraesuis A50 and S. dublin 3246 taken from one calf. In calves 1 and $2, S$. typhimurium strain 4/74 (ST4/74) and its isogenic invH mutant (ST4/74 invH) were used as positive and negative controls respectively as described in the legend of Fig. 1.

Preparation of inocula for invasion assays. Organisms were grown as described by Amin et al. (1994) but were held on ice overnight at $4{ }^{\circ} \mathrm{C}$ in fresh HDB medium to suppress histotoxic activity of $S$. dublin (Bolton et al., 1999). Approximately $10^{9}$ 'stored' organisms were suspended in fresh HDB medium and added to designated $9 \mathrm{~cm}$ loops.

For the one 90 min experiment in pigs, organisms were grown and used fresh without overnight storage at $4{ }^{\circ} \mathrm{C}$; in our recent work (Bolton et al., 1999), S. dublin 3246 organisms grown at $37^{\circ} \mathrm{C}$ and used without storage at $4{ }^{\circ} \mathrm{C}$ were highly histotoxic to rabbit tissues.

Scanning electron microscopy. Biopsies from control and test loops were taken for analysis after $3 \mathrm{~h}$ or after $90 \mathrm{~min}$ bacterial challenge. Tissues were fixed in $2.5 \%$ glutaraldehyde in phosphate buffer for $24 \mathrm{~h}$. Tissues were then dehydrated through a graded acetone series : $50 \%\left(\mathrm{v} / \mathrm{v} \mathrm{H}_{2} \mathrm{O}\right), 70 \%, 90 \%$, $95 \%, 100 \%, 100 \%$ dried (two $15 \mathrm{~min}$ washes for each 

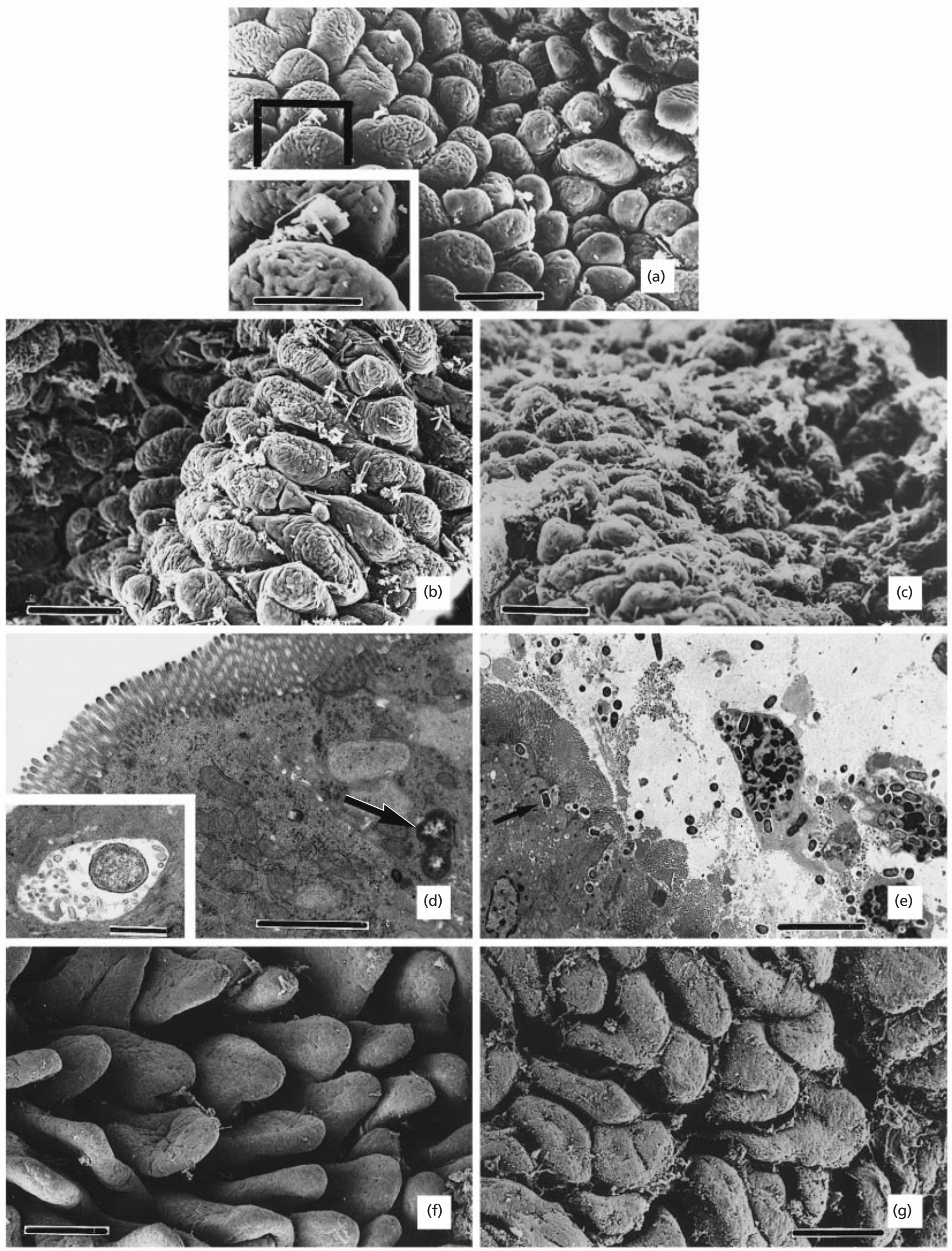

Fig. 3. For legend see facing page. 
acetone concentration). The tissue then underwent criticalpoint drying with liquid $\mathrm{CO}_{2}$ in a Polaron E3000 series II critical-point drier. Pieces of tissue were then mounted on copper stubs with Araldite Rapid (Ciba-Geigy) and sputter coated with Pt in an EMSCOPE SC500 low-voltage sputter coater operated at 100-150 V with 10-20 mA current. Tissues were examined in a JEOL 120 CX II electron microscope with a TEMSCAN attachment. Images were obtained via a secondary electron detector at $40 \mathrm{kV}$.

Transmission electron microscopy (TEM). Biopsies from control and test loops were taken for analysis after $3 \mathrm{~h}$ or after $90 \mathrm{~min}$ bacterial challenge. Tissues were fixed in $2.5 \%$ glutaraldehyde for $1 \mathrm{~h}$ and post-fixed in $1 \% \mathrm{OsO}_{4}$ for $1 \mathrm{~h}$; both the glutaraldehyde and $\mathrm{OsO}_{4}$ solutions were made up in phosphate buffer ( $\mathrm{pH} 7 \cdot 2$; adjusted to $350 \mathrm{mM}$ with sucrose). Tissues were then dehydrated serially in $70 \%, 90 \%$ and $100 \%$ ethanol and embedded in Epon-Araldite (Mollenhauer et al., 1964). Sections (approx. $2 \cdot 5 \mu \mathrm{m}$ thick) were stained with $1 \%$ toluidine blue and observed by light microscopy. Ultra-thin sections (approx. $90 \mathrm{~nm}$ thick) were cut, mounted on Formvarcoated copper grids and stained with methanolic uranyl acetate and Reynold's lead citrate and examined at an accelerating voltage of $60 \mathrm{kV}$ in a JEOL $100 \mathrm{CX}$ II electron microscope.

\section{RESULTS}

\section{Dose-response relationship between inoculum size and number of gentamicin-resistant organisms recovered from $A E$ and $F A E$ in bovine ileal ligated loops challenged with $S$. choleraesuis A50 and S. dublin 3246}

Data from the five calves used in the experiments summarized in Fig. 2 were retrospectively analysed and used to justify the presentation of invasion data for ligated loops as total recoveries of gentamicin-resistant organisms. With each serotype in both $\mathrm{AE}$ and FAE the dose response for inocula ranging from $5 \times 10^{8}$ to $3 \times 10^{9}$ was flat (data not shown), indicating probable saturation of Salmonella binding sites. A similar trend was observed with pigs. This is in contrast to the method used for in vitro assays, in which 'the number of gentamicin-resistant organisms recovered, expressed as a fraction of the initial inoculum' is routinely used as an index of invasiveness (Bolton et al., 1999).

\section{Recoveries of S. dublin and S. choleraesuis from porcine ileal loop mucosae}

In every experiment loops were constructed in a segment of ileum which included both AE and FAE. This was to compare the recoveries of organisms from the two types of mucosae in a competitive situation.

In three pigs $(1,2,3$; Fig. 1a, b), there were clear differences in the recoveries of the non-invasive mutant S. typhimurium $4 / 74 i n v H$ and its invasive parent S. typhimurium 4/74 (used as negative and positive controls respectively), demonstrating that this bioassay in pigs could differentiate between strains which differed in their ability to invade either AE or FAE.

The recoveries of S. dublin 3246 from AE and FAE were comparable to each other; this was also true for S. choleraesuis A50 (Fig. 1a, b).

The recoveries of S. dublin 3246 and S. choleraesuis A50 from porcine AE were comparable to each other; this was also true for FAE (Fig. 1a, b).

In all three pigs, wild-type S. typhimurium 4/74 was more invasive than either S. dublin 3246 or S. choleraesuis A50 (Fig. 1a, b).

\section{Recoveries of S. dublin and S. choleraesuis from bovine ileal loop mucosae}

A similar picture was obtained to that described above for pigs. In two calves (1, 2; Fig. 2a, b), there were clear differences in the recoveries of $S$. typhimurium 4/74 invH and S. typhimurium 4/74, demonstrating that this bioassay in calves could differentiate between strains which differed in their ability to invade either AE or FAE.

In all five calves $(1,2,3,4,5$; Fig. 2a, b), the recoveries of $S$. dublin 3246 from bovine $\mathrm{AE}$ and FAE were comparable to each other; this was also true for $S$. choleraesuis A50.

The recoveries of S. dublin 3246 and S. choleraesuis A50 from bovine AE were comparable to each other; this was also true for FAE (Fig. 2a, b).

Fig. 3. Electron micrographs of porcine tissues exposed to S. dublin 3246 and S. choleraesuis A50. Data in (a-d) are from one $3 \mathrm{~h}$ experiment in one animal but are typical of what was observed in comparable tissues from other animals; organisms were grown at $37^{\circ} \mathrm{C}$, and used after storage at $4{ }^{\circ} \mathrm{C}$. Data in (e-g) are from the one 90 min experiment in one animal in which organisms were grown at $37{ }^{\circ} \mathrm{C}$ and used without storage at $4{ }^{\circ} \mathrm{C}$. (a) Control AE after exposure to HDB medium. The inset shows enlargement of the marked area. Bar, $200 \mu \mathrm{m}$ (inset, $100 \mu \mathrm{m}$ ). (b) S. choleraesuis A50 induced wrinkling of villus tips in AE. Bar, $200 \mu \mathrm{m}$. (c) S. dublin 3246 caused villi in AE to become much less well-defined, shorter and overlaid with debris. Bar, $200 \mu \mathrm{m}$. (d) TEM showed little difference in appearance of S. choleraesuis A50-challenged enterocytes of $A E$ from that of control tissue (not shown). Note the presence of two organisms (with no discernible surrounding vacuolar membrane: arrow) in an otherwise intact-looking cell. Bar, $3 \mu \mathrm{m}$. The inset shows an organism in a vacuole from the 90 min experiment, containing remnants of microvilli. Bar, $1 \mu \mathrm{m}$. (e) AE after 90 min exposure to $S$. dublin 3246. Note the entry of organisms in large numbers into a cell(s) with brush border, the presence in the lumen of damaged bacteria-laden cells, free organisms, and the continued invasion (arrow) of tissue by S. dublin 3246. Bar, $10 \mu \mathrm{m}$. (f) FAE after 90 min exposure to $S$. choleraesuis A50; villi were almost indistinguishable from the control (not shown). Bar, $200 \mu \mathrm{m}$. (g) FAE after 90 min exposure to S. dublin 3246; villi are collapsing, flaccid and losing turgor. Bar, $200 \mu \mathrm{m}$. 
The susceptibility of bovine ileal mucosae to invasion (Fig. 2a, b) seemed to be greater than that of corresponding porcine mucosae (Fig. 1a, b).

\section{Electron microscopic analyses of porcine ileal mucosae after challenge in vivo with $S$. choleraesuis and S. dublin}

Unless otherwise stated, the following data were derived from one $3 \mathrm{~h}$ experiment in one animal but are typical of what was observed in comparable tissues from other animals. Porcine ileal AE appeared normal after exposure to HDB medium (Fig. 3a). S. choleraesuis A50 caused AE villus tips to become more wrinkled, showing classic signs of incipient accelerated cell shedding in response to bacterial invasion (Fig. 3b). Exposure to $S$. dublin 3246 caused greater disruption of AE architecture than did exposure to $S$. choleraesuis A50: villi were much less well-defined, shorter and overlaid with debris (Fig. 3c). The extent of the tissue damage caused by $S$. dublin 3246 was far greater than could be accounted for by the observed presence of organisms. S. choleraesuis A50 organisms were observed in otherwise intactlooking cells, nearly always singly or in small numbers, inside vacuoles of varying size. For example, Fig. 3(d) (main panel) shows two organisms without a discernible surrounding membrane. The inset in Fig. 3(d) (tissue taken after $90 \mathrm{~min}$ in another experiment) shows a typical newly formed membrane-bounded vacuole containing an internalized organism; Fig. 4(f) shows an organism inside a much smaller vacuole.

The following data, unless otherwise stated, were derived from the one 90 min experiment using organisms grown at $37^{\circ} \mathrm{C}$ and used fresh without overnight storage at $4{ }^{\circ} \mathrm{C}$. S. dublin 3246 caused disruption of $\mathrm{AE}$ : damaged bacteria-laden cells and free organisms were seen in the lumen, with continued invasion of microvillus-bearing enterocytes (Fig. 3e). In contrast, even after $3 \mathrm{~h}$, very little epithelial damage was caused by $S$. choleraesuis A50 (Fig. 3d; main panel). After 90 min exposure to $S$. choleraesuis A50 the appearance of FAE was little different from that of control mucosa (Fig. 3f); little additional gross change occurred after $3 \mathrm{~h}$ (not shown). In contrast, after $90 \mathrm{~min}$ S. dublin 3246 caused villi of FAE to collapse, and apparently lose turgor (Fig. 3g).

One of the objectives of this work was to determine the cellular route of entry of Salmonella into porcine gut epithelia. A special search was therefore made for evidence implicating $M$ cells in invasion, particularly of FAE. Under the conditions of our experiments, it was exceedingly difficult to find structures which could be unequivocally identified as $\mathrm{M}$ cells in S. dublinchallenged epithelia: in both the $3 \mathrm{~h}$ experiments with stored organisms and the 90 min experiment with freshly grown organisms, major tissue destruction was induced by this serotype. What is abundantly clear, however, is that, while $S$. dublin organisms may enter porcine gut via $M$ cells, they also enter directly into cells with brush borders. Such entry is neither tissue nor animal specific: S. dublin 3246 could be seen entering microvillus-bearing cells in FAE (Fig. 4a), and enterocytes in AE (see Fig. 3e); these tissues were from two different pigs. Entry into enterocytes was not strain specific: from the $90 \mathrm{~min}$ experiment $S$. dublin 2229 could be seen entering directly into enterocytes (Fig. 4b, c).

In contrast, it was possible to be more definite about $S$. choleraesuis FAE interactions since in both pigs and calves $S$. choleraesuis did not cause the same kind of structural damage as S. dublin. S. choleraesuis A50 could be seen entering $M$ cells (Fig. $4 \mathrm{~d}$, e), and concurrently, directly into enterocytes (Fig. 4e, f). The enterocyte containing the $S$. choleraesuis A50 organism in Fig. 4(f) was four cells removed from the $\mathrm{M}$ cell in Fig. 4(d). From the $90 \mathrm{~min}$ experiment, a similar picture of concurrent entry of $S$. choleraesuis 14/74 into both M cells and enterocytes was obtained (not shown).

\section{Electron microscopic analyses of bovine ileal mucosae after challenge in vivo with $S$. choleraesuis and S. dublin}

A less detailed study of bovine tissues was carried out since the early stages of epithelial invasion had already been thoroughly addressed (Watson et al., 1995; Frost et al., 1997). The picture obtained with bovine gut essentially paralleled that obtained with porcine gut. The following data were derived from one $3 \mathrm{~h}$ experiment in one animal but are typical of what was observed in comparable tissues from other animals.

After $3 \mathrm{~h}$ exposure to $\mathrm{HDB}$ medium, control AE villi were well preserved (Fig. 5a). Exposure to $S$. choleraesuis A50 induced wrinkling of villi, a classic sign of incipient accelerated cell shedding in response to bacterial invasion (Fig. 5b). Organisms were observed inside otherwise intact goblet cells (Fig. $5 \mathrm{~g}$ ) and enterocytes (Fig. 5h). In contrast, villi of AE in loops challenged with S. dublin 3246 were shorter, and some were frankly damaged and overlaid with cellular debris (Fig. 5c).

S. choleraesuis A50 induced relatively minor changes in villus architecture of FAE (Fig. 5d, f) and were not found near to blood vessels. In contrast, S. dublin 3246 caused massive damage to villi in FAE (Fig. 5e); very few intact villi remained and these were highly abnormal (Fig. 5i). However, despite extensive epithelial damage, S. dublin 3246 organisms were found having penetrated to sites adjacent to subepithelial vascular elements (Fig. 5j), indicating that these organisms were highly tissueinvasive as well as histotoxic.

\section{DISCUSSION}

Cultured cells have proved useful for elucidating mechanisms exploited by bacterial pathogens, including Salmonella, in the invasion of eukaryotic cells but are of little value for studying complex bacteria/host interactions in multicellular compartments such as gut. The 

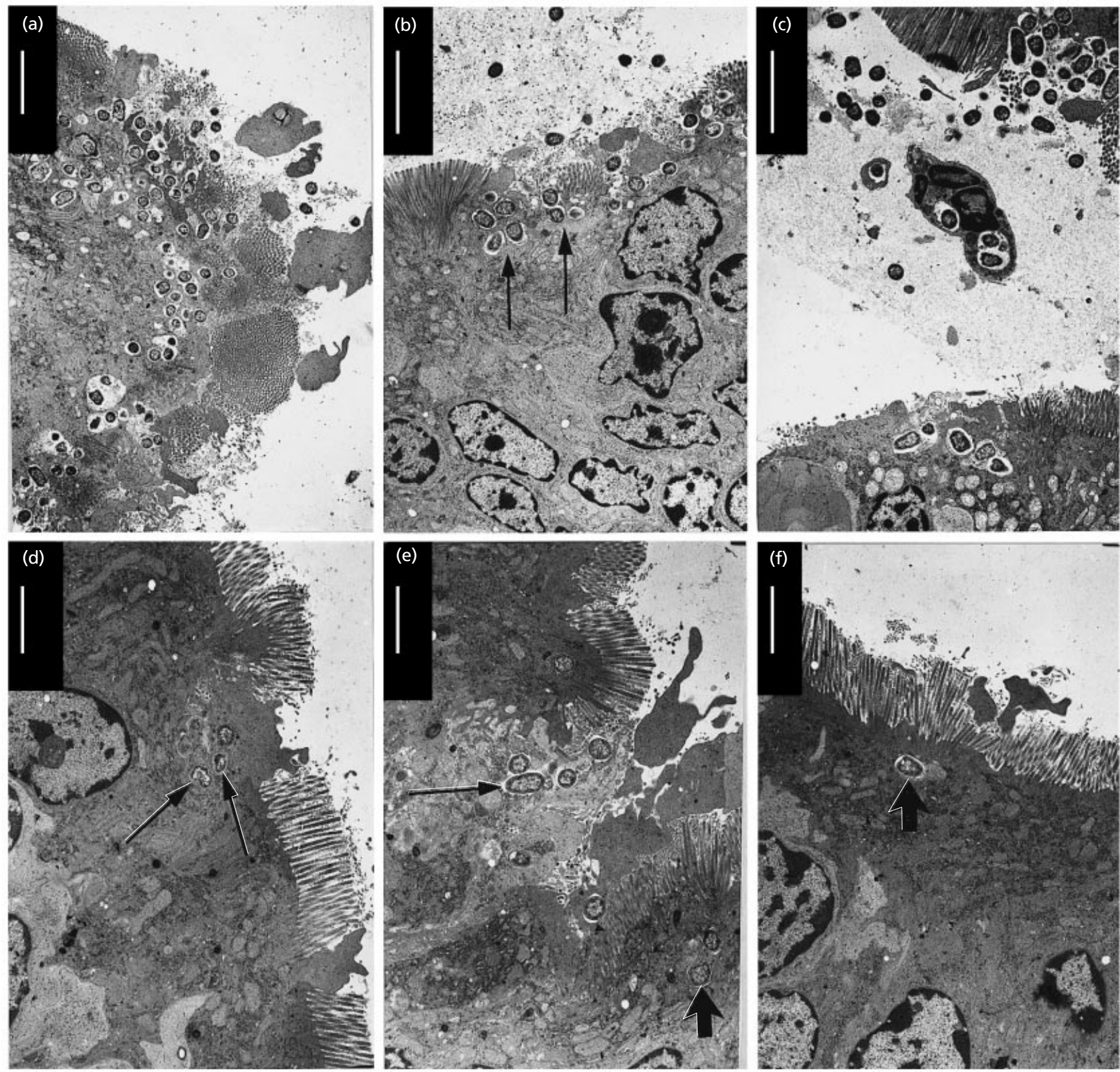

Fig. 4. Electron micrographs of porcine FAE exposed to S. dublin 3246 and S. choleraesuis A50. These data were derived from different experiments as indicated in the text. The micrographs show that both $S$. dublin and $S$. choleraesuis enter porcine epithelia directly via enterocytes in addition to (in the case of $S$. choleraesuis) M cells. (a) A micro-colony of S. dublin 3246 inside enterocytes. Note the disorganized damaged apical brush border. Bar, $4 \mu \mathrm{m}$. (b) S. dublin 2229 invading enterocytes (arrows). Again note the disorganized damaged epithelium. Bar, $4 \mu \mathrm{m}$. (c) Micro-colony of $S$. dublin 2229 directly invading enterocytes (top), and organisms internalized in damaged enterocytes (bottom) and inside an extruded PMN (centre). Bar, $4 \mu \mathrm{m}$. Because of the tissue-destructive properties of S. dublin, it was extremely difficult to find authentic or possible examples of $\mathrm{M}$ cells in these experiments. (d) $\mathrm{S}$. choleraesuis $\mathrm{A} 50$ organisms entering an $\mathrm{M}$ cell (arrows). Bar, $2 \mu \mathrm{m}$. (e). S. choleraesuis A50 organisms inside an $\mathrm{M}$ cell (thin arrow) and a brush-border-bearing enterocyte (thick arrow). Bar, $2 \mu \mathrm{m}$. (f) S. choleraesuis A50 organism inside an otherwise intact enterocyte which was four cells laterally removed from the ' $\mathrm{M}$ ' cell in (e). Bar, $2 \mu \mathrm{m}$.

in vitro organ culture system successfully developed for rabbit tissues (Amin et al., 1994; DeKort et al., 1994; Lodge et al., 1995, 1999; Bolton et al., 1999) proved unsuccessful using porcine and bovine tissues due to failure to maintain mucosal integrity (A. J. Bolton, unpublished observations); hence our resort to ligated ileal loops. The latter model has proved useful for studying interactions between enteric pathogens and 

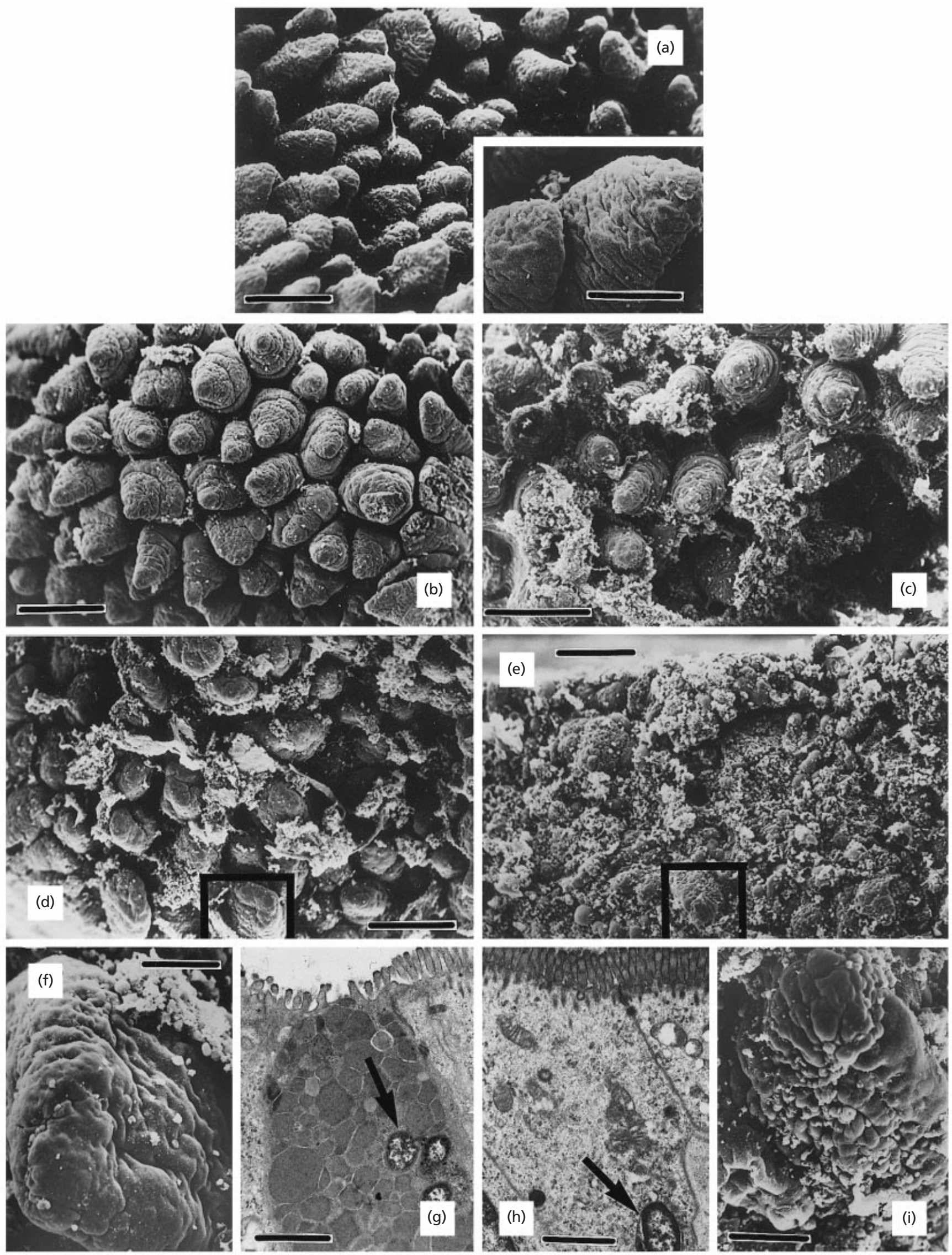

Fig. 5. For legend see facing page. 


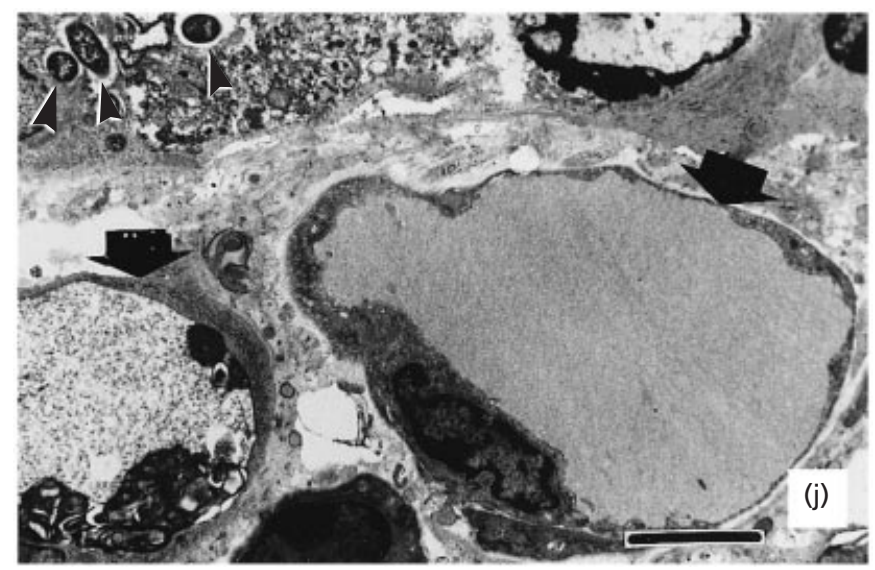

Fig. 5. Electron micrographs of bovine tissues exposed to S. dublin 3246 and S. choleraesuis A50. Data in (a-j) are from one $3 \mathrm{~h}$ experiment in one animal but are typical of what was observed in comparable tissues from other animals. (a) $\mathrm{AE}$ exposed to HDB medium (control). Bar, $200 \mu \mathrm{m}$ (inset, $40 \mu \mathrm{m}$ ). (b) AE exposed to S. choleraesuis A50: villi are more wrinkled, showing classic signs of incipient accelerated cell shedding in response to bacterial invasion. Bar, $200 \mu \mathrm{m}$. (c) $\mathrm{AE}$ exposed to S. dublin 3246: villi are similar to those in (b) but some are frankly damaged and overlaid with cellular debris. Bar, $200 \mu \mathrm{m}$. (d) FAE exposed to S. choleraesuis A50: intact villi are overlaid with largely acellular debris. Bar, $200 \mu \mathrm{m}$. Panel $(\mathrm{f})$ is an enlargement (bar, $40 \mu \mathrm{m}$ ) of the marked area in (d) showing a mainly intact villus (one of many in this typical field). (e) FAE exposed to S. dublin 3246: there were few remaining 'whole' villi and these are collapsing on themselves. Bar, $200 \mu \mathrm{m}$. Panel (i) is an enlargement (bar, $40 \mu \mathrm{m}$ ) of the marked area in (e) showing one of the few remaining severely damaged villus structures. (g) TEM of S. choleraesuis A50 organisms (arrow) inside an intact goblet cell in AE. Bar, $2 \mu \mathrm{m}$. (h) TEM of S. choleraesuis A50 organism (bounded by a tight membrane-bounded vacuole: arrow) in an enterocyte showing only partial damage to mitochondria. Bar, $2 \mu \mathrm{m}$. (j) TEM of $S$. dublin 3246 organisms (black arrowheads) inside a disintegrating cell near to vascular elements (black arrows) in AE. Bar, $4 \mu \mathrm{m}$.

defined regions of intestinal mucosae. Although the introduction of bacteria into closed loops is artificial compared with oral infection of the unobstructed gut, we believe that the level of intestinal invasion taking place in this system is biologically relevant: numbers of bacteria recovered from bovine ileal loops in this study were comparable to those from bovine ileal mucosae $24 \mathrm{~h}$ after oral challenge with strains of S. typhimurium and S. dublin (Watson et al., 1998).

Wild-type virulent $S$. typhimurium $4 / 74$ was recovered (from both porcine and bovine hosts) in approximately 10-fold higher numbers than the attenuated $S$. typhimurium 4/74 strain, indicating that the system could discriminate between highly invasive and noninvasive strains. In the study reported here, S. dublin was recovered from both bovine and porcine ileal mucosae in numbers comparable to $S$. choleraesuis despite inducing more mucosal damage to bovine and porcine ileal mucosae than S. choleraesuis. Therefore, in these experiments, we found no evidence for the idea that the host-restriction exhibited by these serotypes for cattle and pigs is explicable solely in terms of major differences in their respective invasiveness for terminal ileum. However, a double rider must be added. First, it is obvious that the magnitude of mucosal damage must be taken into account when interpreting the biological significance of the data. Second, the dose response around an inoculum size of $10^{9}$ for invasiveness in calves was flat, indicating probable saturation of Salmonella binding sites; we do not know the minimum inoculum sizes which would result in effective invasion of either bovine or porcine ileal epithelia. It would be extremely useful to be able to estimate the numbers of organisms surviving passage through the alimentary tract to distal ileum after oral administration of infectious doses of S. dublin and S. choleraesuis in pigs and calves. Such information could result in more meaningful comparative studies being carried out - in terms of the size and phenotype of challenge inocula - on the invasiveness of host-restricted Salmonella serotypes in pigs and calves. The results of such experiments would either confirm that differential invasiveness of the gut accounts for host restriction or require that explanations for host restriction in systemic Salmonella infections be sought at stages of the complex host/pathogen interaction other than invasiveness for gut epithelia. They would also provide data on the survivability of such strains through the alimentary tract, an unglamorous area that has been completely ignored in this context.

Previously we had shown a correlation between invasiveness for distal rabbit ileum and virulence in human strains of S. typhimurium (Amin et al., 1994; Bolton et al., 1999), which, taken together with this work involving host-restricted strains, suggests the following important points. While all virulent strains of Salmonella are invasive, the converse is apparently not true: invasiveness per se is not a general predictor of Salmonella virulence since the $S$. dublin strains used in this work did not cause clinical disease in pigs when administered orally in doses which routinely infected 
calves (T. S. Wallis, unpublished observations). We have also shown that Salmonella-induced enteritis in rabbits is not due solely to the intestinal invasiveness of salmonellae. Invasive and enteropathogenic strains of $S$. typhimurium never induced fluid secretion in rabbit ileal loops in the absence of a PMN influx, although a PMN influx per se was not responsible for fluid secretion (Wallis et al., 1989). More recently a pathogenicity island (SPI-5) has been identified in S. dublin 2229 and shown to be conserved in a range of different serotypes (Wood et al., 1998). Genes encoded by SPI-5 are involved in S. dublin-induced enteropathogenesis in calves, but not in systemic pathogenesis in mice. Mutation of the SPI-5-associated gene $\operatorname{sop} B$, which encodes a secreted effector protein SopB (an inositol phosphate phosphatase; Norris et al., 1998), significantly reduced (but did not abolish) fluid secretion and PMN influxes, without affecting the invasive phenotype in vivo (Galyov et al., 1997). The enteropathogenicities for calves of S. typhimurium and $S$. dublin strains of equal invasiveness in vivo have also been compared. Strains of S. typhimurium were more enteropathogenic than those of $S$. dublin (Watson et al., 1998), suggesting that the virulence mechanisms of these serotypes may differ. The elucidation of these differences may hold the key to defining host restriction in terms of clinical disease causation rather than in susceptibility to initial infection. Clearly much remains to be unravelled.

There were clear differences in the reaction of both porcine and bovine tissues to the two Salmonella serotypes: S. dublin was clearly more histotoxic than $S$. choleraesuis to both porcine and bovine tissues. We believe the damage to be toxin-mediated, since damage was much more extensive than might have been expected if it were linked to the direct physical presence of bacteria: it was relatively easy to find examples of damage in areas where it was difficult to find attaching/ invading bacteria. If, as would seem highly likely, the damage reported here is mediated by the toxin previously described (Lodge et al., 1999; Bolton et al., 1999), it would follow that the toxin shows no specificity for the tissues of rabbit (Bolton et al., 1999), calf and pig (this work). These experiments suggest that (in the context of gut invasion) there are at least two different biotypes of Salmonella, which cross serotypic boundaries - one which causes rapid initial histotoxic damage to mucosal epithelia, the other which does not. Histotoxic strains will open up additional cellular routes for invasion of gut mucosae by virtue of early damage to gut enterocytes, and denudation of epithelia of enterocytes with subsequent exposure of subepithelial cells and tissues. In addition, the invasiveness of histotoxic strains like S. dublin 3246 will always be underestimated. However, in the context of this work, allowance for this possibility does not provide an explanation of hostrestriction of $S$. dublin in terms of invasiveness: this serotype was both invasive and histotoxic in pigs, calves and rabbits.

The bulk of the work reported here was carried out over $3 \mathrm{~h}$ with organisms grown at $37^{\circ} \mathrm{C}$ and stored on ice at
$4{ }^{\circ} \mathrm{C}$ overnight. This protocol was designed to give a truer measure of the relative intrinsic invasiveness of the strains of $S$. dublin and $S$. choleraesuis by hopefully suppressing the expression of histotoxicity of the former, as was the case for rabbit ileal tissue in vitro (Bolton et al., 1999). Unfortunately it was not possible to carry out a time-based study to measure the rate of initial invasion. As alluded to above, our estimates of S. dublin invasiveness were clearly underestimates since by $3 \mathrm{~h}$ there was evidence of $S$. dublin-induced tissue damage in porcine AE and FAE and particularly in bovine FAE. The limited data from the $90 \mathrm{~min}$ pig experiment, generated with organisms which were grown and used fresh without overnight storage at $4{ }^{\circ} \mathrm{C}$, demonstrates that the expression of overt histotoxicity by $S$. dublin occurs early when an appropriate phenotype is used to inoculate the loops. This observation lends additional weight to the view that definitive experiments on the biological significance of differential invasiveness of these Salmonella serotypes for gut will require the use of 'appropriate in vivo phenotypes'. Tissue destruction by S. dublin is thus not only extensive but rapid, from which it follows that organisms present in gentamicintreated tissues must be intrinsically highly invasive as well as toxigenic. In fact, $S$. dublin organisms were found in deeper tissues and in some cases even near to vascular elements.

Our approach enabled us to compare the relative invasiveness of these S. dublin and S. choleraesuis strains for bovine and porcine ileal mucosae with or without Peyer's patch tissue in a competitive situation in the same loop. At three hours post-infection both serotypes were recovered in comparable numbers from $\mathrm{AE}$ and FAE, confirming and extending previous observations (Frost et al., 1997; Watson et al., 1995) implicating both $\mathrm{AE}$ and FAE in the early stages of the infection process. Clearly, in natural Salmonella/host interactions salmonellae are able to penetrate the apical membranes of $\mathrm{M}$ cells in FAE and also enterocytes in both FAE and AE. This point needs to be emphasized since, as stated in the Introduction, there is now a growing acceptance, based largely on studies in murine ligated loops, that $\mathrm{M}$ cells are the main primary route of Salmonella entry into the gut in vivo. M cells then die, creating a gap in the epithelium through which bacteria can penetrate and then infect adjacent enterocytes via basolateral membranes, resulting in exfoliation of enterocytes, denudation of FAE epithelium and exposure of the basement membrane to luminal bacteria (Jones et al., 1994). It is interesting to note that the damage seen in murine ligated loops $2 \mathrm{~h}$ after infection with very large doses of the mouse-virulent strain S. typhimurium SL 1344 $\left(200 \mu \mathrm{l}\right.$ culture containing approx. $10^{8}$ c.f.u. into a 4-5 cm murine ileal loop; Jones et al., 1994) bears some similarity to that caused by histotoxic $S$. dublin in pigs, calves (this work) and rabbits (Bolton et al., 1999; Lodge et al., 1999). It is possible that the disruption of murine epithelia reported by Jones et al. (1994) was toxin mediated as distinct from being a secondary undefined consequence of $\mathrm{M}$ cell destruction. The 'M cell scenario' 
may hold for S. typhimurium in mice but not necessarily in calves, where both $M$ cells and enterocytes were infected very early in infection (Frost et al., 1997; Watson et al., 1995). Infection of $\mathrm{M}$ cells in bovine FAE preceded the initiation of independent infection of enterocytes in $\mathrm{AE}$ by only $10 \mathrm{~min}$. It is also manifestly not true that $\mathrm{M}$ cells are the major route of invasion in rabbits, in which we have used large numbers of ligated ileal loops (Wallis et al., 1986) each one deliberately constructed in segments of gut free of Peyer's patches, something which is very easy to do by virtue of the physical distribution of Peyer's patches in rabbit small intestine. It was logistically not possible for us to conduct a series of time-course observations with pigs similar to what had already been done with calves. Hence we cannot make definitive statements about the detailed kinetics of infection of different cell types. However, the point we make from this work is that one can demonstrate concurrent internalization of nonhistotoxic $S$. choleraesuis by enterocytes physically removed from active foci of $M$ cell penetration in porcine ileal epithelia. The biological significance of interactions between S. typhimurium and M cells may well depend on the precise Salmonella serotype (or strain)/host combination.

As with S. typhimurium TML (Worton et al., 1989), S. dublin was nearly always seen entering the gut in microcolonies; in sharp contrast, S. choleraesuis was observed nearly always entering as single organisms or in groups of two or three. The entry by S. typhimurium and $S$. dublin as microcolonies is not due to clumping, since it is not difficult to prepare single cell suspensions for use in challenging gut either in vivo or in vitro. It is a highly reproducible but an as yet unexplained phenomenon which might be significant in initiating infection and an example of bacterial co-operative activity.

\section{ACKNOWLEDGEMENTS}

We thank Sue M. Paulin for assistance with surgical procedures. We (J.S. and M.P.O.) thank the BBSRC and MAFF (T.S.W.) for grant support for this work.

\section{REFERENCES}

Amin, I. I., Douce, G. R., Osborne, M. P. \& Stephen, J. (1994). Quantitative studies of invasion of rabbit ileal mucosa by Salmonella typhimurium strains which differ in virulence in a model of gastroenteritis. Infect Immun 62, 569-578.

Bolton, A. J., Martin, G. D., Osborne, M. P., Wallis, T. S. \& Stephen, J. (1999). Invasiveness of Salmonella typhimurium, Salmonella choleraesuis and Salmonella dublin for rabbit terminal ileum in vitro. J Med Microbiol 48, 800-810.

Clark, M. A., Jepson, M. A., Simmons, N. L. \& Hirst, B. H. (1994). Preferential interaction of Salmonella typhimurium with mouse Peyers Patch M cells. Res Microbiol 145, 543-552.

Clark, M. A., Reed, K. A., Lodge, J., Stephen, J., Hirst, B. H. \& Jepson, M. A. (1996). Invasion of murine intestinal $M$ cells by Salmonella typhimurium inv mutants severely deficient for invasion of cultured cells. Infect Immun 64, 4363-4368.
DeKort, G., Bolton, A., Martin, G. D., Stephen, J. \& Vandeklundert, J. A. M. (1994). Invasion of rabbit ileal tissue by Enterobacter cloacae varies with the concentration of OmpX in the outer membrane. Infect Immun 62, 4722-4726.

Frost, A. J., Bland, A. P. \& Wallis, T. S. (1997). The early dynamic response of the calf ileal epithelium to Salmonella typhimurium. Vet Pathol 34, 369-386.

Galyov, E. E., Wood, M. W., Rosqvist, R., Mullan, P. B., Watson, P. R., Hedges, S. \& Wallis, T. S. (1997). A secreted effector protein of Salmonella dublin is translocated into eukaryotic cells and mediates inflammation and fluid secretion in infected ileal mucosa. Mol Microbiol 25, 903-912.

Jones, B. D., Ghori, N. \& Falkow, S. (1994). Salmonella typhimurium initiates murine infection by penetrating and destroying the specialized epithelial M cells of the Peyers Patches. J Exp Med 180, 15-23.

Lodge, J., Douce, G. R., Amin, I. I., Bolton, A. J., Martin, G. D., Chatfield, S., Dougan, G., Brown, N. L \& Stephen, J. (1995). Biological and genetic characterization of TnphoA mutants of Salmonella typhimurium TML in the context of gastroenteritis. Infect Immun 63, 762-769.

Lodge, J., Bolton, A. J., Martin, G. D., Osborne, M. P., Ketley, J. M. \& Stephen, J. (1999). A Salmonella histotoxin. J Med Microbiol 48, 811-818.

Mollenhauer, H. H. (1964). Plastic embedding mixture for use in electron microscopy. Stain Technol 39, 111-114.

Norris, F. A., Wilson, M. P., Wallis, T. S., Galyov, E. E. \& Majerus, P. W. (1998). SopB, a protein required for virulence of Salmonella dublin, is an inositol phosphate phosphatase. Proc Natl Acad Sci USA 95, 14057-14059.

Penheiter, K. L., Mathur, N., Giles, D., Fahlen, T. \& Jones, B. D. (1997). Non-invasive Salmonella typhimurium mutants are avirulent because of an inability to enter and destroy $M$ cells of ileal Peyer's patches. Mol Microbiol 24, 697-709.

Wallis, T. S., Starkey, W. G., Stephen, J., Haddon, S. J., Osborne, M. P. \& Candy, D. C. A. (1986). The nature and role of mucosal damage in relation to Salmonella typhimurium-induced fluid secretion in the rabbit ileum. J Med Microbiol 22, 39-49.

Wallis, T. S., Hawker, R. J. H., Candy, D. C. A., Qi, G. M., Clarke, G. J., Worton, K. J., Osborne, M. P. \& Stephen, J. (1989). Quantification of the leukocyte influx into rabbit ileal loops induced by strains of Salmonella typhimurium of different virulence. J Med Microbiol 30, 149-156.

Watson, P. R., Paulin, S. M., Bland, A. P., Jones, P. W. \& Wallis, T. S. (1995). Characterization of intestinal invasion by Salmonella typhimurium and Salmonella dublin and effect of a mutation in the invH gene. Infect Immun 63, 2743-2754.

Watson, P. R., Galyov, E. E., Paulin, S. M., Jones, P. W. \& Wallis, T. S. (1998). Mutation of invH, but not stn, reduces Salmonellainduced enteritis in cattle. Infect Immun 66, 1432-1438.

Wood, M. W., Jones, M. A., Watson, P. R., Hedges, S., Wallis, T. S. \& Galyov, E. E. (1998). Identification of a pathogenicity island required for Salmonella enteropathogenicity. Mol Microbiol 29, 883-891.

Worton, K. J., Candy, D. C. A., Wallis, T. S., Clarke, G. J., Osborne, M. P., Haddon, S. J. \& Stephen, J. (1989). Studies on early association of Salmonella typhimurium with intestinal mucosa in vivo and in vitro: relationship to virulence. J Med Microbiol 29, 283-294.

Received 13 January 1999; revised 7 May 1999; accepted 9 June 1999. 